\title{
Lytic polysaccharide monooxygenases (LPMOs) facilitate cellulose nanofibrils production
}

\author{
Céline Moreau', Sandra Tapin-Lingua², Sacha Grisel ${ }^{3}$, Isabelle Gimbert ${ }^{3}$, Sophie Le Gall' , Valérie Meyer ${ }^{4}$, \\ Michel Petit-Conil ${ }^{4}$, Jean-Guy Berrin ${ }^{3}$, Bernard Cathala ${ }^{1}$ and Ana Villares ${ }^{1 *}$ (D)
}

\begin{abstract}
Background: Lytic polysaccharide monooxygenases (LPMOs) are copper-dependent enzymes that cleave polysaccharides through an oxidative mechanism. These enzymes are major contributors to the recycling of carbon in nature and are currently used in the biorefinery industry. LPMOs are commonly used in synergy with cellulases to enhance biomass deconstruction. However, there are few examples of the use of monocomponent LPMOs as a tool for celIulose fibrillation. In this work, we took advantage of the LPMO action to facilitate disruption of wood cellulose fibers as a strategy to produce nanofibrillated cellulose (NFC).
\end{abstract}

Results: The fungal LPMO from AA9 family (PaLPMO9E) was used in this study as it displays high specificity toward cellulose and its recombinant production in bioreactor is easily upscalable. The treatment of birchwood fibers with $\mathrm{PaLPMO9E}$ resulted in the release of a mixture of $\mathrm{C} 1$-oxidized oligosaccharides without any apparent modification in fiber morphology and dimensions. The subsequent mechanical shearing disintegrated the LPMO-pretreated samples yielding nanoscale cellulose elements. Their gel-like aspect and nanometric dimensions demonstrated that LPMOs disrupt the cellulose structure and facilitate the production of NFC.

Conclusions: This study demonstrates the potential use of LPMOs as a pretreatment in the NFC production process. LPMOs weaken fiber cohesion and facilitate fiber disruption while maintaining the crystallinity of cellulose.

Keywords: Lytic polysaccharide monooxygenase (LPMO), Biorefinery, Nanofibrillated cellulose (NFC), Microfluidizer, Molar mass, 13 C CP/MAS NMR

\section{Background}

Replacement of the fossil oil-based products with renewable materials is a critical demand to implement bioeconomy. Cellulose appears as a potential candidate since it is the most abundant renewable polymer produced on Earth through photosynthesis [1,2]. Since a few decades, interest for cellulose-based materials has tremendously increased by the renewal of nanocelluloses [3-8]. The term nanocellulose is employed when the cellulosic objects present at least one dimension in the nanoscale

\footnotetext{
*Correspondence: ana.villares@inra.fr

1 UR1268 Biopolymères Interactions Assemblages, INRA, 44316 Nantes, France

Full list of author information is available at the end of the article
}

(1-100 nm). Nanocelluloses are usually divided into two main types: cellulose nanocrystals (CNCs) and nanofibrillated cellulose (NFC). CNCs have been reported for the first time in the 1950s and are commonly obtained by acid hydrolysis resulting in nanorods with high aspect ratio derived from the crystalline part of the fibers $[9$, 10]. NFC is obtained by mechanical delamination of the fibers as firstly reported in the early 1980s. It consists in flexible fibrils with length higher than $\mathrm{CNCs}$ and lateral dimensions depending on the production process but ranging from few nanometers to tens of nanometers [11, 12]. Both CNC and NFC are used in many applications thanks to their amazing properties such as high mechanical strength, ability to stabilize emulsions, gas barrier, dispersing properties and others [6, 7, 13-17]. Methods 
of NFC production from wood cellulosic fibers are numerous but most of them can be summarized in two main steps [8]. The first one consists in a pretreatment that can be either chemical (by 2,2,6,6-tetramethylpiperidine-1-oxyl (TEMPO)-mediated oxidation or carboxymethylation) or enzymatic (endoglucanase and/or xylanase treatment) [18-25]. Mechanical nanofibrillation is achieved in the second step by different technologies such as high-pressure homogenization, microfluidization or grinding, among the most widely reported $[6,8]$. The pretreatment step is mandatory since starting fibers cannot be otherwise processed. The goal of the pretreatment step is to (i) weaken the fiber cohesion to allow fiber processing, (ii) lower the energy consumption of the mechanical process and (iii) improve the final quality of NFC. Thus, the efficiency of the pretreatment step is a key point in the process since it greatly impacts the quality of the final NFC and also the energy efficiency of the process. Accordingly, the development of innovative pretreatments for NFC production is still an intense field of investigation.

Lytic polysaccharide monooxygenases (LPMOs) are copper-dependent enzymes that cleave polysaccharides through an oxidative mechanism. They are classified as Auxiliary Activity (AA) enzymes by the CarbohydrateActive enZymes database (CAZy; http://www.cazy.org). The proposed mechanism of action consists of the cleavage of cellulose by the insertion of oxygen at $\mathrm{C} 1$ and/or $\mathrm{C} 4$, with the subsequent formation of a lactone, which is spontaneously hydrolyzed to aldonic acid or a ketoaldose, respectively [26]. These enzymes have been initially used in synergy with glycoside hydrolases to boost the saccharification of plant biomass, and they have been incorporated in last-generation commercial enzyme cocktails for the production of biofuels [27]. Recent studies have shown that LPMOs may be used in synergy with cellulases and/or xylanases to facilitate the deconstruction of cellulose fibers in an attempt to produce NFC $[28,29]$. In that line of research, we had previously demonstrated that monocomponent LPMOs can disrupt the cellulose fibers structure by the creation of nicking points that weaken the fiber cohesion [30]. In this study, we assessed the use of LPMOs as pretreatment of cellulosic fibers for NFC production. Bleached birchwood Kraft pulp was submitted to LPMO action and then processed with a microfluidizer to demonstrate the industrial relevance of the process. LPMO-treated fibers were successfully dispersed at nanoscale while untreated fibers could not be processed. The structure of the NFC all along the dispersion treatment was investigated by using MorFi analysis, optical and atomic force microscopy and solid-state NMR. Monosaccharide composition of the fibers and molar mass distribution of cellulose chains were also determined. Results provide new insights on the LPMO action as well as the proof of concept that LPMOs can be efficiently implemented for NFC production.

\section{Results \\ Production of PaLPMO9E in bioreactor}

An important feature to consider upon production of recombinant LPMOs is the perfect processing of the signal peptide during secretion to ensure correct binding of the catalytic copper ion by the histidine brace that includes the $\mathrm{N}$-terminal histidine residue. Optimal processing of signal peptides during heterologous production is protein dependent, and heterogeneity on $\mathrm{N}$-terminal sequences is a recurrent problem [31]. In $P$. pastoris, the use of the $\alpha$-mating factor $(\alpha-\mathrm{MF})$ as signal peptide is sometimes associated with incorrect cleavage by the Ste13 protease [31]. Therefore, we designed a plasmid construct using the native signal sequence of the PaLPMO9E encoding gene to foster recombinant protein production in P. pastoris. Using this strategy, the production yield of $\mathrm{PaLPMO}$ E, assessed by measuring the recombinant protein production in the medium after induction, significantly increased (about twofold) when the native signal sequence was used. Beyond the fact that the recombinant LPMO was expressed to high level, $\mathrm{N}$-terminal sequencing indicated that the processing of the signal peptide was fully achieved yielding a functional enzyme batch.

Taking advantage of this new construct, we set up the recombinant production of PaLPMO9E in bioreactor up to $2 \mathrm{~g}$ of protein per liter of culture after 5 days (Additional file 1: Fig. S1) enabling the enzymatic processing of wood cellulosic fibers under industrially relevant conditions.

\section{LPMO pretreatment allows the production of NFC}

Nanofibrillated cellulose (NFC) was produced according to the work plan described in Fig. 1. Bleached birchwood Kraft fibers $(28 \mathrm{~g})$ at a consistency of $3.5 \%(\mathrm{w} / \mathrm{w})$ were incubated with $\mathrm{PaLPMO9E}$ and ascorbate as electron donor. Ascorbate was set at $0.5 \mathrm{mM}$, which is slightly lower in concentration than previously reported $[30,32]$. However, taking into account the pulp consistency, the ascorbate/LPMO ratio is similar to our previous experiments. After $24 \mathrm{~h}$ of incubation, fibers were boiled for $10 \mathrm{~min}$ to inactivate the enzymes and then filtered and dispersed at $2 \%$ consistency (Additional file 1: Fig. S2). Firstly, supernatants of the PaLPMO9Etreated fibers were analyzed by high performance anion exchange chromatography (HPAEC) for the detection of soluble non-oxidized and oxidized oligosaccharides released upon enzymatic action. Oxidized oligomers were detected, and the degree of polymerization 


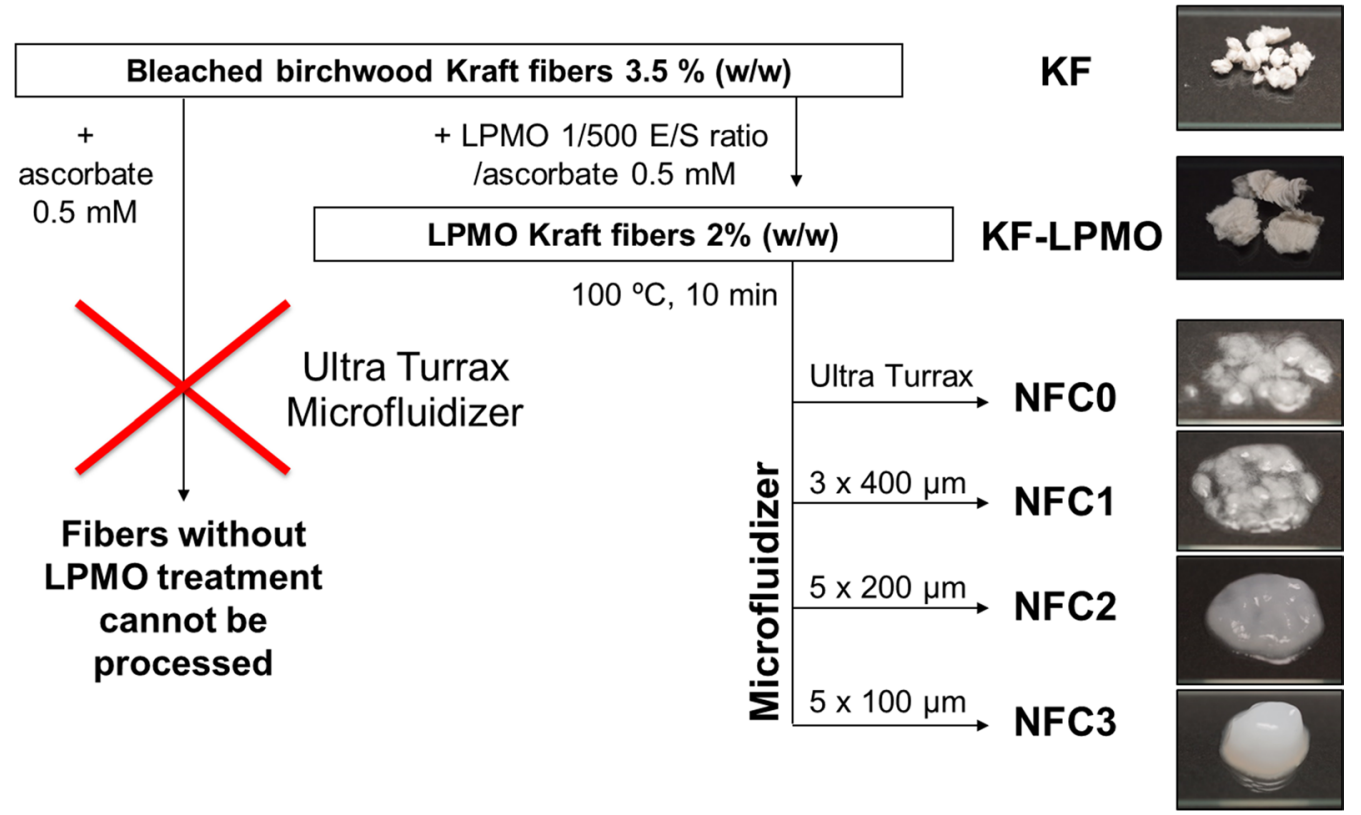

Fig. 1 General scheme of the treatments performed on bleached birchwood Kraft fibers for the preparation of nanofibrillated cellulose (NFC) by PaLPMO9E pretreatment combined with mechanical shearing and photographs of the samples

(DP) ranged between DP2 and DP6 (Additional file 1: Fig S3), as previously observed for the action of the enzyme PaLPMO9E on phosphoric acid swollen cellulose (PASC) [32]. Only C1-oxidized oligomers and not $\mathrm{C} 4$ oxidation products were detected confirming that PaLPMO9E is active on cellulose substrate with a C1-type regioselectivity. After filtration and mild dispersion by an Ultra Turrax device, higher mechanical shearing was achieved by using a microfluidizer processor, which is commonly used for NFC production at industrial scale. The microfluidizer is equipped of three Z-chambers that progressively decrease in diameter $(400,200$ and $100 \mu \mathrm{m})$ to increase shearing and fibrillation efficiency. The first significant result of this study was that LPMO-pretreated fibers were able to be processed in the microfluidizer without further mechanical pretreatment, which is the first requirement for the production of NFC from cellulose fibers. In contrary, control fibers that had undergone pretreatment in the same conditions but without enzyme loading could not be homogenized, as they blocked the system at the entrance to the cell. Figure 1 shows the photographs of bleached birchwood Kraft fibers throughout the treatments. LPMO-treated suspensions obtained after passing through the $200 \mu \mathrm{m}$ chamber (NFC2) displayed gel consistency, which is an indirect proof of efficient dispersion at nanoscale. This effect was more pronounced in the case of NFC3 (passing through the $100 \mu \mathrm{m}$ chamber).

\section{LPMO-treated fibers are efficiently nanofibrillated by microfluidization}

Optical microscopy images from starting bleached birchwood Kraft fibers mostly consisted of dispersed fibers of tens of micrometers in width and several hundreds of micrometers in length, which are the typical dimensions expected (Fig. 2). Upon PaLPMO9E treatment, fibers still remained as bundles that seemed to be slightly fibrillated even if width did not change significantly. After microfluidization, fibrillation was clear for all samples. The first passes in the $\mathrm{Z}$ chamber of $400 \mu \mathrm{m}$ (NFC1) produced the rupture of several bundles resulting in a slight decrease in width $(13.4 \pm 4.4 \mathrm{~mm})$ and the release of small fragments that were scarcely detected by optical microscopy. The second passes through the $\mathrm{Z}$ chamber of $200 \mu \mathrm{m}$ (NFC2) resulted in a clear decrease in both width and length of fibers, so that bundles were separated and cut in length. This effect was even more noticeable after the third treatment through the $100 \mu \mathrm{m}$ chamber (NFC3), which produced a clear disruption of fibers. In this case, sample was completely fibrillated and a homogeneous gel-like matrix could be observed. Observations at the nanoscale by AFM supported these assumptions (Fig. 2). Starting birchwood Kraft fibers (KF) 


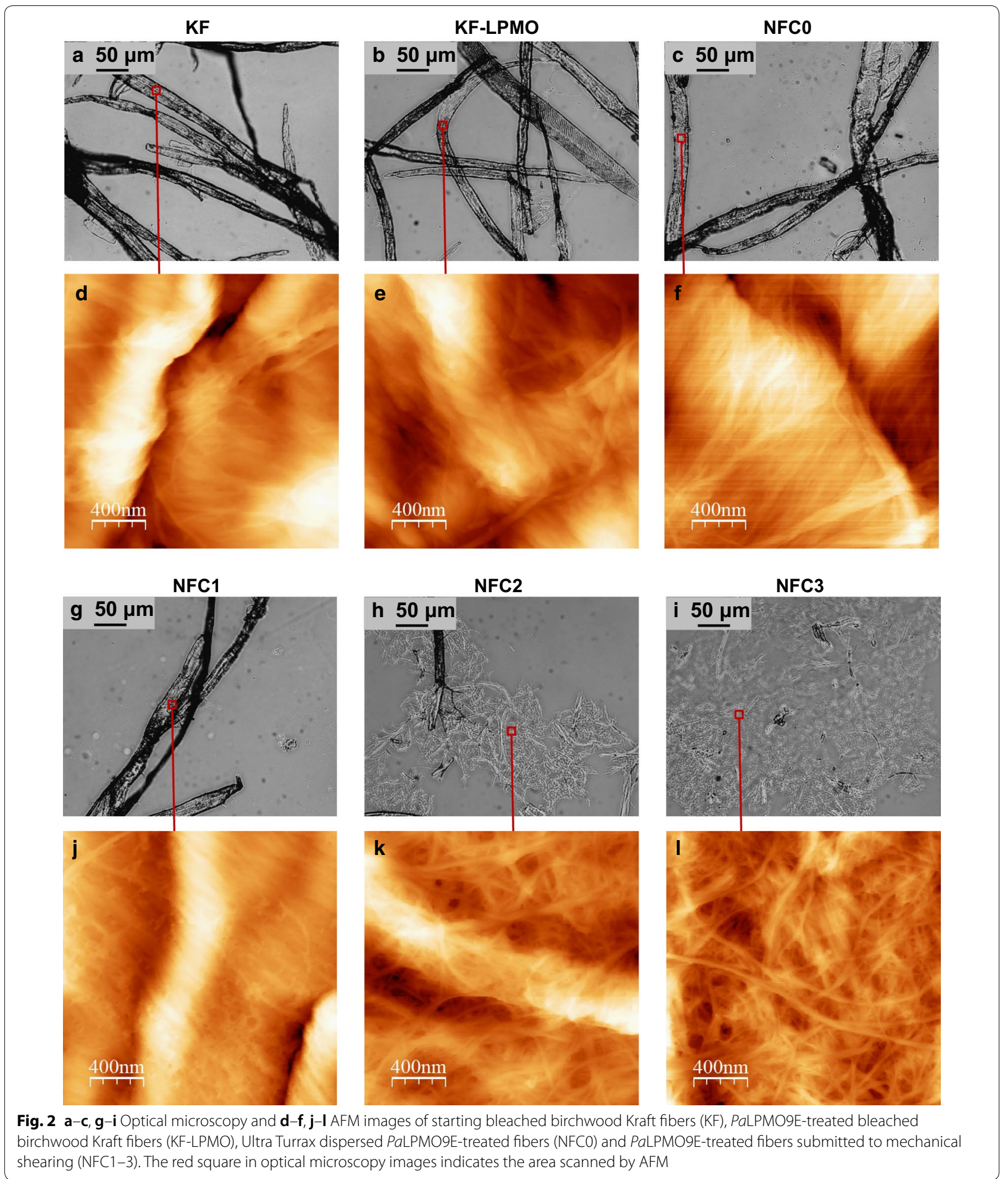

showed the particular pattern of wrinkles and microfibrils more or less aligned, characteristic of cellulose fibers [33]. After PaLPMO9E treatment (KF-LPMO), AFM images showed a slight separation of the microfibrils, even if the fiber structure remained preserved. The effect of $\mathrm{PaLPMO9E}$ was confirmed by the AFM images after 
dispersion by Ultra Turrax (NFC0), where more defined microfibrillated structures were observed. Even if optical microscopy images did not detect significant changes in the fiber structure, AFM suggested that PaLPMO9E action resulted in the separation of microfibrils. When samples were submitted to the first shearing mechanical treatment $(400 \mu \mathrm{m}, \mathrm{NFC1})$, the fiber structure was still maintained; however, the passage through the $200 \mu \mathrm{m}$ chamber (NFC2) provoked the disintegration of the fiber, with the subsequent release of nanofibrillated cellulose. This effect was more pronounced after the passage through the $100 \mu \mathrm{m}$ chamber (NFC3), where the images clearly showed an entangled network of nanofibrillated cellulose.

Optical and AFM images provided useful information but could be incomplete since they address only a portion of the sample. Thus, global assessment of the distribution pattern in the samples was determined by the MorFi analyzer. This analysis, with a detection threshold of $15 \mu \mathrm{m}$, does not characterize the smallest generated elements and nanoparticles but provides a size distribution of the residual fiber elements. The mean area-weighted length (Fig. 3) did not change significantly upon $\mathrm{PaLPMO9E}$ treatment, or even after the passage through the $400 \mu \mathrm{m}$ vessel (NFC1), while suspensions obtained after passing by the $200 \mu \mathrm{m}$ vessels were highly microfibrillated (NFC2), as their gel consistency suggested. Then, higher fibrillation was obtained by performing a series of homogenization in the $100 \mu \mathrm{m}$ chamber (NFC3). However, if we compare to similar treatments using endoglucanases or exoglucanases treatments at $0.1-1 \%$ loading followed by mechanical shearing, the lengths of fibers and the percentages of fine elements obtained in this work were still very coarse [34]. Usually, for the same working pulp pretreated by mechanical enzymatic pretreatment


Fig. 3 Geometrical characteristics (mean area-weighted length and percentage of fine content) of starting bleached birchwood Kraft fibers (KF), PaLPMO9E-treated bleached birchwood Kraft fibers (KF-LPMO), Ultra Turrax dispersed PaLPMO9E-treated fibers (NFC0) and PaLPMO9E-treated fibers submitted to mechanical shearing (NFC1-3)

Table 1 Monosaccharide composition on the percent dry weight basis of starting bleached birchwood Kraft fibers (KF), PaLPMO9E-treated bleached birchwood Kraft fibers (KF-LPMO), Ultra Turrax dispersed PaLPMO9E-treated fibers (NFC0) and PaLPMO9E-treated fibers submitted to mechanical shearing (NFC1-3)

\begin{tabular}{|c|c|c|c|c|c|c|}
\hline Sample & Rha & Ara & Xyl & Man & Glc & Inacc. cell \\
\hline $\mathrm{KF}$ & $0.5 \pm 0.0$ & $0.2 \pm 0.1$ & $23.7 \pm 0.5$ & $1.6 \pm 1.0$ & $74.1 \pm 0.6$ & $70.2 \pm 1.0$ \\
\hline KF-LPMO & $0.4 \pm 0.1$ & $0.1 \pm 0.0$ & $23.8 \pm 0.6$ & $1.2 \pm 0.5$ & $74.4 \pm 0.3$ & $70.3 \pm 0.3$ \\
\hline NFCO & $0.3 \pm 0.1$ & $0.2 \pm 0.2$ & $23.6 \pm 0.1$ & $1.1 \pm 0.1$ & $74.8 \pm 0.3$ & $70.1 \pm 0.2$ \\
\hline $\mathrm{NFC1}$ & $0.4 \pm 0.0$ & $0.5 \pm 0.1$ & $23.9 \pm 0.3$ & $1.2 \pm 0.0$ & $74.0 \pm 0.4$ & $69.0 \pm 0.6$ \\
\hline $\mathrm{NFC2}$ & $0.1 \pm 0.1$ & $0.3 \pm 0.2$ & $23.2 \pm 0.3$ & $0.8 \pm 0.1$ & $75.6 \pm 0.5$ & $69.6 \pm 0.5$ \\
\hline NFC3 & $0.4 \pm 0.2$ & $0.2 \pm 0.2$ & $23.1 \pm 0.2$ & $1.2 \pm 0.3$ & $75.2 \pm 0.4$ & $66.8 \pm 0.4$ \\
\hline
\end{tabular}

Results are expressed as the mean percentage \pm standard deviation 
and homogenized with a $100 \mu \mathrm{m}$ vessel, the suspensions have $\sim 90 \%$ of fine elements (elements of size less than $80 \mu \mathrm{m}$ ) compared to $83 \%$ in the case of the pulp pretreated by LPMOs (NFC3).

\section{Monosaccharide composition is not affected by LPMO and mechanical treatments}

The effect of both PaLPMO9E and mechanical treatments was assessed by the determination of the monosaccharide composition of the samples throughout the process. Sample compositions are reported in Table 1.

The major sugars recovered were glucose, xylose and small amounts of arabinose, galactose and rhamnose, as expected for hardwood delignified fibers containing cellulose and xylan as major polymers [35]. The major conclusion from the sugar analysis was that the monosaccharide profile did not change along the fractionation process neither after the PaLPMO9E treatment nor during microfluidization. Other useful information that can be obtained from the monosaccharide determination assay is the amount of accessible and inaccessible cellulose fractions. Indeed, samples can be submitted either to a harsh hydrolysis that releases the total monosaccharides or to a milder acid hydrolysis that is usually recognized to release only the accessible sugar fractions (i.e., mostly from hemicellulose and amorphous cellulose) [36]. Even though this analysis remains questionable for the real structural features of polymers released, it gives valuable qualitative information in the case of samples submitted to stepwise modification such as in the present case. From the results, it appeared that the inaccessible fraction proportions (Table 1, last column) remained stable for the first three samples (KF, KF-LPMO and NFC0), suggesting that the PaLPMO9E treatment did not change the major architecture of the fibers and likely the crystalline organization. During the mechanical treatment (NFC1-3), the values decreased slightly probably due to nanofibrillation and associated higher specific surface. Nevertheless, this decrease remained limited suggesting the retention of high crystallinity, which will be later confirmed by solid-state NMR.

\section{Molar mass distribution of cellulose chains is decreased by the mechanical treatment}

Dissolution of untreated and PaLPMO9E-treated samples was successfully achieved by using the solvent system dimethyl acetamide (DMAc)/lithium chloride $9 \%$ $(w / w)$ followed by elution in DMAc/LiCl $0.9 \%$. The dissolution procedure used was inspired by the standardized protocol proposed by Potthast et al. [37], and the recovery of cellulose was in all the cases higher than $65 \%$ and even in some cases close to $85 \%$, ensuring the

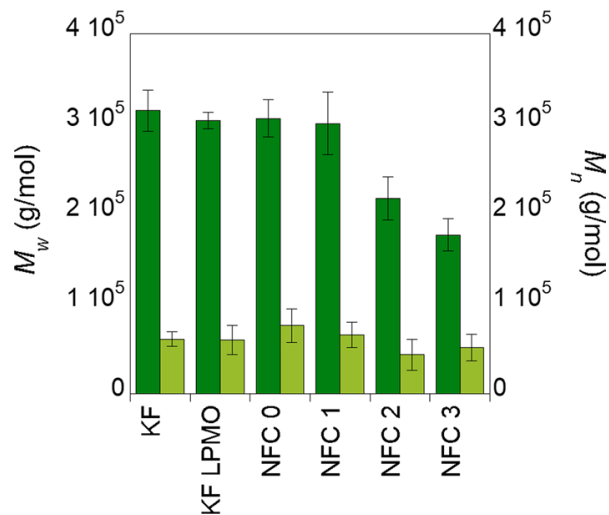

Fig. 4 Weight average molar mass $\left(M_{w}\right)$ and number average molar mass $\left(M_{n}\right)$ of starting bleached birchwood Kraft fibers (KF), PaLPMO9E-treated bleached birchwood Kraft fibers (KF-LPMO), Ultra Turrax dispersed PaLPMO9E-treated fibers (NFCO) and PaLPMO9E-treated fibers submitted to mechanical shearing (NFC1-3)

reliability of the determination. Molar mass was determined by multi-angle laser light scattering (MALLS) as absolute molar mass determination detector and by a differential refractometer (RI) as concentration detector. The values of the weight average molar mass $\left(M_{\mathrm{w}}\right)$ and number average molar mass $\left(M_{\mathrm{n}}\right)$ and representative RI traces are reported in Fig. 4 and Additional file 1: Fig. S4, respectively. The values obtained for KF and KFLPMO (i.e., samples before mechanical treatment) indicated that after the PaLPMO9E treatment, only a very small decrease of the $M_{\mathrm{w}}$ was observed $\left(31510^{5} \mathrm{~g} \mathrm{~mol}^{-1}\right.$ for KF vs $30310^{5} \mathrm{~g} \mathrm{~mol}^{-1}$ for KF-LPMO) while the $M_{\mathrm{n}}$ values remained constant. This result seemed to indicate that PaLPMO9E induced some cleavage of the cellulose chains but this observation needs to be strengthened by future studies since the difference observed was lower than the dispersion of the values. Concerning the mechanically treated samples, $M_{\mathrm{w}}$ values of NFCO and NFC1 samples were identical to KF-LPMO (lower than KF) while the NFC2 and NFC3 showed a clear decrease. In all cases, $M_{\mathrm{n}}$ values remained stable within the error bar interval. The recovery yield decreased along the treatment. Hence, it ranged about $85 \%$ for KF, then decreased around $70 \%$ for KF-LPMO, NFC0 and NFC1 and finally reached $60-65 \%$ for the two last samples (NFC2 and NFC3). This fact could indicate the formation of nanometric elements that might be removed during the solvent exchange procedure or that display limited solubility as previously demonstrated [38]. 


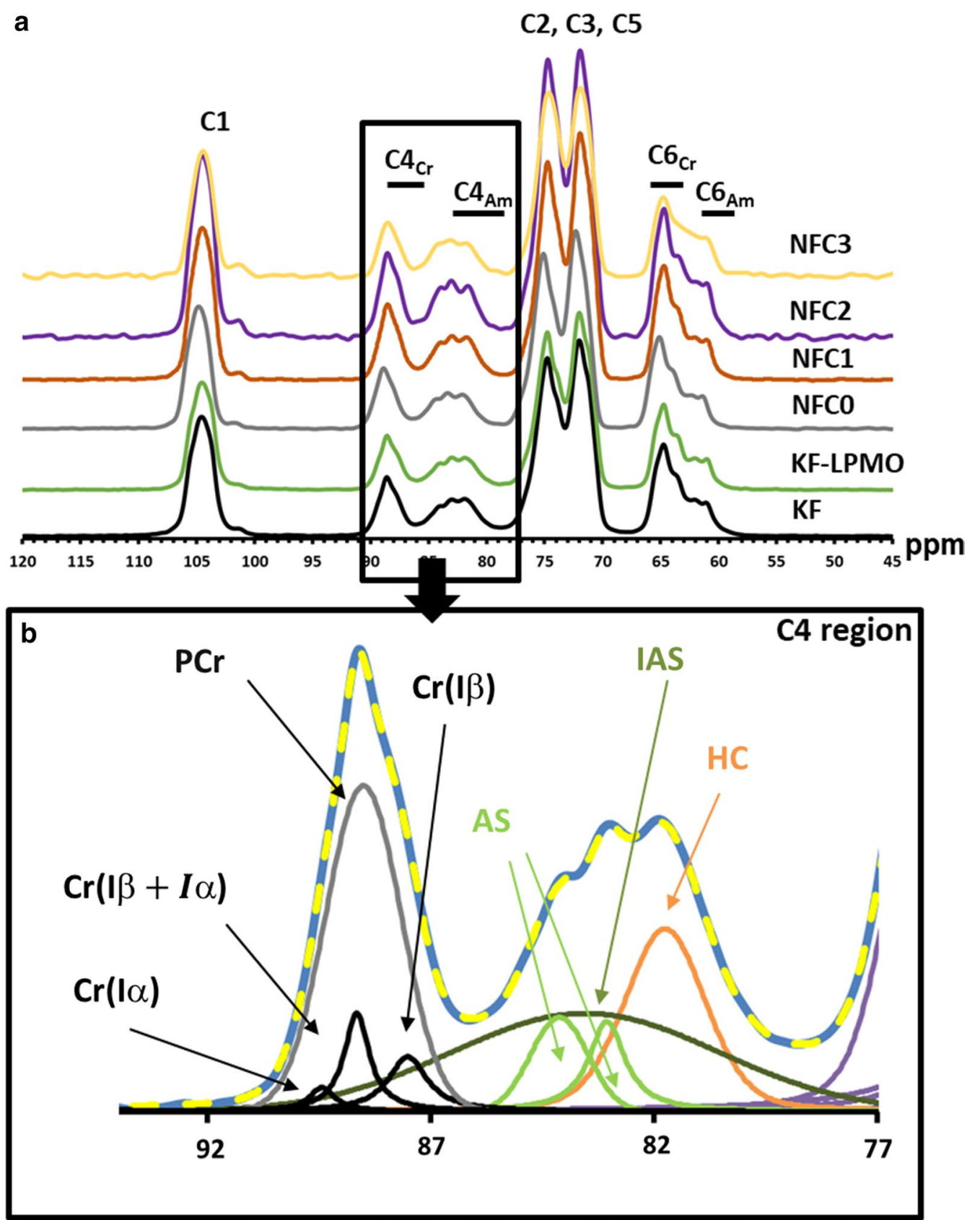

Fig. 5 a Solid-state ${ }^{13} \mathrm{C}$ CP/MAS NMR spectra of starting bleached birchwood Kraft fibers (KF, black line), PaLPMO9E-treated bleached birchwood Kraft fibers (KF-LPMO, green line), Ultra Turrax dispersed PaLPMO9E-treated fibers (NFCO, gray line) and PaLPMO9E-treated fibers submitted to mechanical shearing by the $400 \mu \mathrm{m}$ chamber (NFC1, red line), by the $200 \mu \mathrm{m}$ chamber (NFC2, purple line), and by the $100 \mu \mathrm{m}$ chamber (NFC3, yellow line). The frame part of the NMR spectra corresponds to the $C 4$ region. $\mathbf{b}$ Typical deconvolution of the $C 4$ region from ${ }^{13} \mathrm{C} \mathrm{CP/MAS} \mathrm{NMR}$ spectrum of the KF sample. $\mathrm{Cr}(\mathrm{la}), \mathrm{Cr}(\mathrm{I} \beta)$ and $\mathrm{Cr}(\mathrm{la}+\beta)$ correspond to crystalline cellulose forms; $\mathrm{PCr}$ corresponds to para-crystalline contribution; AS/IAS corresponds to accessible/inaccessible surfaces in the amorphous part of cellulose; and $\mathrm{HC}$ to hemicellulose contribution

LPMOs trigger elementary fibrils separation but do not decrease the nanofibers crystallinity

To investigate deep changes induced in the fiber structure, solid-state ${ }^{13} \mathrm{C} \mathrm{CP} / \mathrm{MAS} \mathrm{NMR}$ spectroscopy was used to analyze cellulose fibers before and after the
PaLPMO9E action and mechanical treatments. The dif-

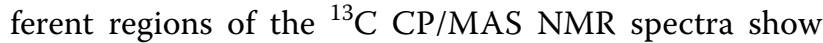
the typical distinct signals of $\mathrm{C} 1$ ( $\delta$ 98-108 ppm), C4 ( $\delta 78-92 \mathrm{ppm}), \mathrm{C} 2, \mathrm{C} 3, \mathrm{C} 5$ carbons $(\delta 68-78 \mathrm{ppm})$ and C6 $(\delta 58-68 \mathrm{ppm})$ from cellulose (Fig. 5a). The most 
informative region in the NMR spectra of cellulose is the C4 region between 78 and $92 \mathrm{ppm}$, which contains sharp signals ranging from 86 to $92 \mathrm{ppm}$ corresponding to $\mathrm{C} 4$ carbons situated in crystalline cellulose domains $\left(\mathrm{C}_{\mathrm{Cr}}\right.$ in Fig. 5a). The $\mathrm{C} 4$ carbons of more disordered regions (or amorphous domains) are distributed in a broad band ranging from 78 to $86 \mathrm{ppm}\left(\mathrm{C}_{\mathrm{am}}\right.$, Fig. $\left.5 \mathrm{a}\right)$. The ${ }^{13} \mathrm{C} \mathrm{CP} /$ MAS spectra of all samples were deconvoluted by fitting C4-region according to the NMR fitting procedure previously reported $[30,39,40]$. Typically, fitted lines of the C4 ( $\delta 78-92 \mathrm{ppm})$ are shown in Fig. 5b with four peaks, $\mathrm{Cr}(\mathrm{I} \alpha), \mathrm{Cr}(\mathrm{I} \beta)$ and $\mathrm{Cr}(\mathrm{I} \alpha+\beta)$ corresponding to crystalline cellulose forms, together with para-crystalline $(\mathrm{PCr})$ cellulose. For the non-crystalline cellulose domain, three peaks were detected: a pair of signals at 83 and $84 \mathrm{ppm}$ assigned to two non-equivalent sites at the accessible crystallite surfaces (AS) and a very broad signal at 83.4 ppm assigned to inaccessible cellulose surface (IAS). The broad peak located at $\sim 81.4 \mathrm{ppm}$ in the $\mathrm{C} 4$ region was assigned to hemicellulose and more specifically to xylan in accordance with the monosaccharide composition. From the fitting data, the accessible (AS)/total fibril (AS + IAS) surface ratio was determined for all samples as well as the cellulose crystallinity index (CrI) defined as the peak area ratio of four lines for the crystalline part (i.e., $\mathrm{Cr}(\mathrm{I} \alpha), \mathrm{Cr}(\mathrm{I} \beta)$ and $\mathrm{Cr}(\mathrm{I} \alpha+\beta)$ and $\mathrm{PCr})$ and seven lines for all the cellulose $C-4$ region [40, 41]. All results are summarized in Table 2.

The degree of cellulose crystallinity was similar to previous values obtained for pulps of birch containing hemicelluloses [42]. Concerning the PaLPMO9E and mechanical treatments, $\mathrm{CrI}$ index was found to be rather stable for all samples. No change was observed after the PaLPMO9E treatment, and only a slight reduction was observed after the passage through the $200 \mu \mathrm{m}$ chamber (NFC2). The hemicellulose content was also determined, and results were consistent with the monosaccharide composition; it was found to be stable around
$20 \%$ suggesting that neither the $P a$ LPMO9E action nor the mechanical disintegration induced a hemicellulose removal. Lateral fibril (LFD) and fibril aggregate (LFAD) dimensions were also determined [43]. Concerning LFD, calculated values were also found stable at about $2.2 \mathrm{~nm}$, slightly lower than values reported for other cellulosic fibers [30, 43, 44]. In contrary, LFAD decreased during the treatment from $19 \mathrm{~nm}$, found for starting birchwood fibers, down to $9 \mathrm{~nm}$ for the PaLPMO9E-treated and mechanically disintegrated (NFC3) samples. Nevertheless, the major decrease was observed during the mechanical treatment and not after the PaLPMO9E action, and it was more remarkable as the chamber diameter was reduced. Therefore, the accessible surface increased during all treatments, which confirmed the separation of fibrils at nanoscale. This finding was in good agreement with AFM images that showed mechanical delamination and the formation of individual nanofibers upon the effect of PaLPMO9E and mechanical shearing.

\section{Discussion \\ LPMO action allows efficient nanofibrillation of cellulosic fibers}

In this work, we used a monocomponent LPMO enzyme system as a pretreatment of cellulose fibers for the NFC production. We submitted bleached Kraft pulp fibers to the action of the fungal PaLPMO9E and further mechanical treatment by microfluidizer. Currently, for any production of NFCs from bleached Kraft fibers, it is necessary to carry out a pretreatment prior to homogenization in order to reduce the fibers size and to allow them to pass through the homogenizer without clogging the chambers. Presently, at industrial scale, the most commonly used pretreatments are chemical or enzymatic. Chemical pretreatments, such as TEMPO-mediated oxidation or carboxymethylation, introduce negative charges on the fiber surface inducing electrostatic repulsion that improves the disintegration of cellulose fibers $[18,19$,

Table 2 Values of crystallinity index (Crl), hemicellulose percentage (\%HC), lateral fibril dimensions (LFD) and lateral fibril aggregate dimensions (LFAD) and accessible/total fibril surface ratio (AS/(AS + IAS)), calculated from the C4-region deconvolution of the solid-state ${ }^{13} \mathrm{C}$ CP/MAS NMR spectra from the starting bleached birchwood Kraft fibers (KF), PaLPMO9E-treated bleached birchwood Kraft fibers (KF-LPMO), Ultra Turrax dispersed PaLPMO9E-treated fibers (NFC0) and PaLPMO9E-treated fibers submitted to mechanical shearing (NFC1-3)

\begin{tabular}{|c|c|c|c|c|c|}
\hline Sample & Crl (\%) & HC (\%) & $\mathrm{LFD}^{\mathrm{a}}(\mathrm{nm})$ & $\operatorname{LFAD}^{a}(\mathrm{~nm})$ & $A S /(A S+I A S)$ \\
\hline $\mathrm{KF}$ & $50.28 \pm 2.99$ & $22.42 \pm 2.39$ & $2.27 \pm 0.13$ & $19.05 \pm 4.43$ & $24.18 \pm 4.38$ \\
\hline KF-LPMO & $49.94 \pm 1.54$ & $18.97 \pm 2.23$ & $2.20 \pm 0.07$ & $18.00 \pm 2.18$ & $24.86 \pm 2.29$ \\
\hline NFCO & $51.13 \pm 0.93$ & $19.12 \pm 0.52$ & $2.23 \pm 0.93$ & $15.14 \pm 0.42$ & $29.67 \pm 0.42$ \\
\hline $\mathrm{NFC1}$ & $53.71 \pm 0.02$ & $19.82 \pm 1.35$ & $2.12 \pm 0.06$ & $14.69 \pm 2.39$ & $32.77 \pm 2.39$ \\
\hline NFC2 & $47.88 \pm 0.02$ & $18.85 \pm 2.34$ & $2.39 \pm 0.12$ & $11.29 \pm 0.40$ & $36.73 \pm 0.40$ \\
\hline NFC3 & $47.65 \pm 0.80$ & $20.19 \pm 1.17$ & $2.39 \pm 0.03$ & $9.33 \pm 0.43$ & $43.86 \pm 0.43$ \\
\hline
\end{tabular}

a Assuming a factor of $0.57 \mathrm{~nm}$ 
45]. Enzymatic pretreatments are usually achieved by the action of endoglucanases either alone or in synergy with other enzymes in order to facilitate the refining process treatment [18-25].

Lytic polysaccharide monooxygenase enzymes appear as a novel strategy for facilitating fibrillation of cellulose fibers. In previous studies, we had demonstrated that the enzyme PaLPMO9H induces modifications on the cellulose network that trigger the disintegration of fibers and facilitate the formation of nanofibers [30, 46]. Even using a mild mechanical treatment consisting of dispersion and sonication, cellulose fibers were disrupted down to nanoscale. Subsequent studies performed by Valenzuela et al. [28] investigated the synergy between bacterial LPMOs and endoglucanases followed by high-pressure homogenization for the production of NFC from flax pulp. The authors found significantly higher yields when both enzymes were combined (24.3\%) compared to pure LPMO (12.7\%) or monocomponent endoglucanase (17.0\%) alone. Yields are far from that reported for chemical pretreatments of flax fibers, such as TEMPO at a concentration of $5 \mathrm{mg}$ per gram of cellulose (yield about $70 \%)[47,48]$, which could be ascribed to the content of hemicelluloses that hinder the fibrillation process. $\mathrm{Hu}$ et al. [29] used a combination of endoglucanase, LPMO and xylanases to enhance nanofibrillation of bleached Kraft pulp. The authors demonstrated that the addition of xylanase favors the cleavage of cellulose chains by LPMO and endoglucanases [49].

In this work, the first and most important result is that the PaLPMO9E-treated fibers can be efficiently processed without any clogging of the devices while the reference fibers that were not PaLPMO9E-treated blocked the microfluidizer. Even if no change is observed in size and morphology of birchwood fibers after PaLPMO9E treatment, mechanical shearing clearly revealed the efficient action of PaLPMO9E. The passage through the $400 \mu \mathrm{m}$ chamber produced a slight decrease in fiber length; however, it was the passage through $200 \mu \mathrm{m}$ which induced fibrillation of the fibers and a global decrease of length and diameter. Similarly to common enzymatic treatments (endoglucanase and/or exoglucanase), pure PaLPMO9E disrupted birchwood fibers to the nanometric size, as shown using AFM. Siqueira et al. [34] observed that exoglucanases preserved the network structure of cellulose, whereas the action of endoglucanases resulted in a mixture of nanofibers and nanocrystals. In our study, the action of $\mathrm{PaLPMO}$ E yielded a pulp with a rather uniform nanofibril composition that potentially reduces the need for mechanical refining [50].

\section{LPMO action mechanism assumption}

Beside the demonstration of the efficiency of PaLPMO9E to produce NFC, the aim of our work was also to provide new insights in the LPMO mechanism of action on the cellulosic fibers. The fibers structure was thus investigated by various techniques leading to the following three main features: (i) PaLPMO9E cleaved the cellulosic chains as demonstrated by the HPAEC results. Nevertheless, the cleavage was somehow limited since the amount of oxidized oligomers was low. HPSEC analysis led to similar conclusions since a small decrease in molar mass was observed after the PaLPMO9E treatment for KF-LPMO, NFC0 and NFC1 samples, even if these observations need to be further investigated. Nevertheless, this small variation could be associated with a decrease in the recovery yield. One possible explanation is the formation of nanometric fractions that might be lost during solvent exchange, suggesting that the PaLPMO9E action occurred in the outer surface of the fibers protecting the inner cellulosic chains. (ii) PaLPMO9E did not directly induce nanofibrils disruption since all microscopy techniques and ${ }^{13} \mathrm{C} \mathrm{CP} / \mathrm{MAS}$ NMR indicated that nanofibrillation occurred when the diameter of the $\mathrm{Z}$ chamber was reduced to $200 \mu \mathrm{m}$ and not before (e.g., not after the PaLPMO9E treatment). HPSEC data confirmed this trend since a drastic decrease of $M_{\mathrm{w}}$ was observed for the NFC2 sample compared to NFC1, indicating that the weakening of the fibers boosts mechanical action to reduce the chain length. (iii) PaLPMO9E did not change the fibers crystallinity as indicated directly by the ${ }^{13} \mathrm{C} \mathrm{CP} /$ MAS NMR results and indirectly by the monosaccharide composition.

The combination of these results leads to the conclusion that PaLPMO9E likely acts at the surface of the elementary fibril through an oxidative cleavage that releases only few short soluble oligomers (lower than $0.1 \%$ of the fibers mass), while the major part of the chains remains stacked together. In fact, in the model of cellulose arrangement [43], cellulose fibrils are mainly associated as a part of aggregates, which means that a high proportion of the fibril surface area and crystallinity regions are inside aggregates, preventing direct enzymatic attack. Thus, the inner part of the elementary fibers seems to be preserved after PaLPMO9E action keeping crystallinity and molar mass nearly intact. Nevertheless, mechanical treatment provides enough energy to disrupt the PaLPMO9E-treated cellulose network inducing a clear weakening of the elementary fiber cohesion and increasing the accessible surface. 


\section{Conclusions}

This work describes for the first time the action of a single LPMO followed by mechanical shearing as a strategy for the fabrication of nanofibrillated cellulose (NFC). The treatment of birchwood fibers by PaLPMO9E alone did not modify fiber cohesion and morphology, and fiber dimensions seemed to remain unchanged before mechanical treatment. Nevertheless, the use of mechanical shearing by a microfluidizer processor induced fibrillation and nanometer-sized fibrils were obtained. The use of LPMO enzymes not only allowed the passage of cellulose fibers through the microfluidizer chamber, but also resulted in a homogeneous distribution of nano-size fibrils that maintained fiber crystallinity. This enzymatic process is upscalable as the production of recombinant LPMO was set up in bioreactor.

\section{Materials and methods PaLPMO9E production}

The codon-optimized DNA sequence (Genebank ID CAP67740) encoding was previously synthesized as described in Bennati-Granier et al. [32]. In this study, an alternative construct where the yeast $\alpha$-MF from the expression vector $\mathrm{PPICZ}-\alpha$ was swapped with the native signal peptide was designed. The PmeI-linearized plasmid was used for transformation into electrocompetent P. pastoris X33 cells as described previously [51]. Zeocinresistant $P$. pastoris transformants were then screened for optimal protein production. The best-producing transformants were grown in $1 \mathrm{~L}$ of BMGY containing $1 \mathrm{~mL} \mathrm{~L}^{-1}$ of $\mathrm{PTM}_{4}$ salts $\left(2 \mathrm{~g} \mathrm{~L}^{-1} \mathrm{CuSO}_{4} \cdot 5 \mathrm{H}_{2} \mathrm{O} ; 3 \mathrm{~g}\right.$ $\mathrm{L}^{-1} \mathrm{MnSO}_{4} \cdot \mathrm{H}_{2} \mathrm{O} ; 0.2 \mathrm{~g} \mathrm{~L}^{-1} \mathrm{Na}_{2} \mathrm{MoO}_{4} \cdot 2 \mathrm{H}_{2} \mathrm{O} ; 0.02 \mathrm{~g} \mathrm{~L}^{-1}$ $\mathrm{H}_{3} \mathrm{BO}_{3} ; 0.5 \mathrm{~g} \mathrm{~L}^{-1} \mathrm{CaSO}_{4} \cdot 2 \mathrm{H}_{2} \mathrm{O} ; 0.5 \mathrm{~g} \mathrm{~L}^{-1} \mathrm{CoCl}_{2}$; $12.5 \mathrm{~g} \mathrm{~L}^{-1} \mathrm{ZnSO}_{4} \cdot 7 \mathrm{H}_{2} \mathrm{O} ; 22 \mathrm{~g} \mathrm{~L}^{-1} \mathrm{FeSO}_{4} \cdot 7 \mathrm{H}_{2} \mathrm{O}$; biotin $0.2 \mathrm{~g} \mathrm{~L}^{-1}$; concentrated $\mathrm{H}_{2} \mathrm{SO}_{4} 1 \mathrm{~mL}$ ) in shaken flasks at $30{ }^{\circ} \mathrm{C}$ in an orbital shaker $(200 \mathrm{rpm})$ for $16 \mathrm{~h}$ to an $\mathrm{OD}_{600}$ of 2-6. Expression was induced by transferring the cells into $200 \mathrm{~mL}$ of BMMY containing $1 \mathrm{ml} \mathrm{L}^{-1}$ of $\mathrm{PTM}_{4}$ salts at $20^{\circ} \mathrm{C}$ in an orbital shaker $(200 \mathrm{rpm})$ for another 3 days. Each day the medium was supplemented with $3 \%(\mathrm{v} / \mathrm{v})$ methanol.

Bioreactor production of PaLPMO9E was carried out in 1.3-L New Brunswick BioFlo 115 fermentors (Eppendorf, Hamburg, Germany) following the P. pastoris fermentation process guidelines (Invitrogen) as described in Couturier et al. [39]. Recombinant enzyme was secreted up to $\sim 2 \mathrm{~g} \mathrm{~L}^{-1}$ (Additional file 1: Figs. S1, S2).

\section{Purification of PaLPMO9E}

The culture supernatants were recovered by pelleting the cells by centrifugation at $2700 \mathrm{~g}$ for $5 \mathrm{~min}, 4^{\circ} \mathrm{C}$ and filtered on $0.45 \mu \mathrm{m}$ filters (Millipore, Molsheim, France) to remove any remaining cells. After adjusting the $\mathrm{pH}$ to 7.8 , the supernatants were filtered once more on $0.2 \mu \mathrm{m}$ filters and loaded onto $5 \mathrm{~mL}$ Histrap columns (GE healthcare, Buc, France) connected to an Akta Xpress system (GE healthcare). Prior to loading, the columns were equilibrated in Tris $\mathrm{HCl} 50 \mathrm{mM}$ pH 7.8 and $\mathrm{NaCl} 150 \mathrm{mM}$ (buffer A). The loaded columns were then washed with five column volumes $(\mathrm{CV})$ of $10 \mathrm{mM}$ imidazole in buffer $\mathrm{A}$, before the elution step with $5 \mathrm{CV}$ of $150 \mathrm{mM}$ imidazole in buffer A. After elution, the fractions containing the purified proteins were pooled and buffer was exchanged to Tris $\mathrm{HCl} \mathrm{pH} 7.8, \mathrm{NaCl} 50 \mathrm{mM}$ using PD-10 columns (GE Healthcare). An aliquot of each fraction was loaded onto an SDS-PAGE stain-free gel (Bio-rad, Marnes-la-Coquette, France) to check protein purity. Protein concentration was determined with a Nanodrop ND-2000 spectrophotometer (Thermo Fisher Scientific, IL, USA) using the theoretical mass and the molar extinction coefficient calculated from the protein sequence.

\section{Cellulosic fibers}

A commercial bleached birchwood Kraft pulp from Stora Enso was used.

\section{LPMO treatment and NFC production}

All procedure steps of bleached birchwood Kraft fibers (KF) through enzymatic treatment and homogenization procedures are summarized in Fig. 1. Enzymatic treatment of cellulosic fibers ( $28 \mathrm{~g}$ in total) with PaLPMO9E was carried out in a Tornado multiple chamber reactor (Additional file 1: Fig. S2). Enzymatic treatment was run in parallel in four reaction chambers in order to assess reproducibility. Enzymatic reaction was performed in sodium acetate buffer ( $50 \mathrm{mM}, \mathrm{pH} 4.8)$ in the presence of ascorbic acid at $0.5 \mathrm{mM}$ using an enzyme/substrate ratio of $1: 500$ with a $3.5 \%(w / v)$ consistency during $24 \mathrm{~h}$ under constant stirring and at $50{ }^{\circ} \mathrm{C}$. The enzymatic reaction was stopped by boiling for $10 \mathrm{~min}$, and the wet cake was obtained by filtration and washing (Additional file 1: Fig. S2). Fibers were redispersed at $2 \%$ concentration $(w / w)$ in water (KF-LPMO). The suspensions obtained were homogenized using an Ultra Turrax T25 homogenizer for $30 \mathrm{~s}$ at $7500 \mathrm{rpm}$ (short time to improve fiber dispersion without cutting) (NFC0). The suspensions obtained were then homogenized through a M-110 EH-30 microfluidizer processor (Microfluidics, USA) at a concentration of $2 \%$ ( $w / w$, dry matter content). The slurry was passed through a piston pump that applies a high pressure. This microfluidizer has three Z-shaped interaction chambers with internal diameters of 400, 200 and $100 \mu \mathrm{m}$, and it allows working at a constant flow rate of about

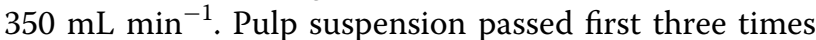
through the $400 \mu \mathrm{m}$ chamber with operating pressure 
of 100 bar (NFC1), then five times through a chamber of $200 \mu \mathrm{m}$, where the operating pressure was $1500 \mathrm{bar}$ (NFC2), and finally five times through the $100 \mu \mathrm{m}$ chamber operating at 2000 bar (NFC3).

\section{Analysis of the soluble sugars by HPAEC-PAD}

The oxidized and non-oxidized oligosaccharides generated after action of PaLPMO9E on bleached birchwood Kraft fibers were analyzed by high-performance anion exchange chromatography coupled with pulsed amperometric detection (HPAEC-PAD) (Thermo Fisher Scientific, Waltham, USA) as described by Westereng et al. [52] using non-oxidized cello-oligosaccharides as standards (Megazyme, Wicklow, Ireland). Oligosaccharides standards oxidized at the $\mathrm{C} 1$ position were produced from non-oxidized cello-oligosaccharides using a cellobiose dehydrogenase as described in Bennati-Granier et al. (31).

\section{Morphological analysis of the fibers}

The morphological characteristics of the fibers contained in the suspensions (starting bleached birchwood Kraft fibers or LPMO-pretreated fibers) were determined by passing through the MorFi analyzer (Techpap, France), based on optics and flow cell measurement.

\section{Optical and atomic force microscopy}

Cellulose fibers were deposited onto freshly cut mica substrates from fiber solutions at $0.1 \mathrm{~g} \mathrm{~L}^{-1}$ and dry overnight. Fibers were observed by an Olympus IX51 microscope with a $20 \times$ objective. Atomic force microscopy imaging was performed with the cantilever located on certain cellulose fibers with the aid of an optical microscope. Topographical images were registered by a Catalyst AFM (Bruker). The images were obtained in tapping mode under ambient air conditions (temperature and relative humidity) using a monolithic silicon tip (ScanasystAir, Bruker) with a spring constant of $0.4 \mathrm{~N} \mathrm{~m}^{-1}$ and a nominal frequency of $70 \mathrm{kHz}$. Image processing was performed with the WSxM 4.0 software [53].

\section{Monosaccharide composition determination}

Individual neutral sugar composition of cellulosic fibers was identified and quantified after sulfuric acid degradation [36] as their alditol acetates derivatives by gas-liquid chromatography (GC) [54]. For total monosaccharides determination, $5 \mathrm{mg}$ of dried cellulosic sample was firstly prehydrolyzed by $13 \mathrm{M}$ sulfuric acid for $1 \mathrm{~h}$ at $30{ }^{\circ} \mathrm{C}$ and then hydrolyzed in $1 \mathrm{M}$ sulfuric acid for $2 \mathrm{~h}$ at $100{ }^{\circ} \mathrm{C}$. For accessible monosaccharide determination, soluble fractions were hydrolyzed by $2 \mathrm{M}$ TFA at $120^{\circ} \mathrm{C}$ for $2 \mathrm{~h}$. GC analysis was performed with a TG-225 GC column $\left(30 \times 0.32 \mathrm{~mm}\right.$ ID) using a TRACE ${ }^{\mathrm{TM}}$ Ultra Gas
Chromatograph (Thermo Scientific ${ }^{\mathrm{TM}}$; temperature $205^{\circ} \mathrm{C}$. carrier gas $\mathrm{H}_{2}$ ). Standard sugars solution and inositol as internal standard were used for calibration. All sample analyses were done in triplicate.

\section{High performance size exclusion chromatography (HPSEC)} About 80-100 mg of cellulose samples was weighted and dispersed in water $(0.1 \% w t)$ during one night under vigorous stirring. Fibers were then filtered through $0.45 \mu \mathrm{m}$ PTFE membranes. The fiber cake was then redispersed three times in anhydrous methanol (50 mL each time) followed by three additional redispersions in anhydrous dimethylacetamide $(50 \mathrm{~mL})$. Then, the DMAc fibers swollen cake was added to 5 or $10 \mathrm{~mL}$ of $\mathrm{DMAc} / \mathrm{LiCl}$ (9\% w/w) under mechanical stirring during $24 \mathrm{~h}$ before tenfold dilution with anhydrous DMAc. The solution was then filtered and injected on a size exclusion chromatography system (OMNISEC Resolve, Malvern) with $N, N$-dimethylacetamide/lithium chloride $(0.9 \% \mathrm{w} / \mathrm{v})$ as the eluent. The SEC columns used were Viscotec Tguard, LT4000L, LT5000L and LT7000L. The system was equipped with a multi-angle laser light scattering Malvern SEC-MALS 20 and OMNISEC Reveal devices (Malvern). Calculations were performed with a $d n / d c$ value of $0.136 \mathrm{~mL} \mathrm{~g}^{-1}$ and performed using OMNISEC software.

\section{Cross-polarization/magic angle spinning (CP/MAS) NMR}

For NMR analysis, samples $(100 \mathrm{mg})$ were rehydrated in $50 \mu \mathrm{L} \mathrm{H} \mathrm{H}_{2} \mathrm{O}$ and water excess was absorbed using an adsorbent. About 80-100 mg of each sample was packed into $4 \mathrm{~mm}$ NMR rotor. All cross-polarization magic angle (CP/MAS) NMR experiments were acquired on a Bruker Avance III 400 spectrometer operating at a ${ }^{13} \mathrm{C}$ frequency of $100.62 \mathrm{MHz}$ equipped with a double-resonance $\mathrm{H} / \mathrm{X}$ $\mathrm{CP} / \mathrm{MAS} 4 \mathrm{~mm}$ probe. Measurements were conducted at room temperature with a MAS spinning rate of $9 \mathrm{kHz}$. The CP pulse sequence parameters were $3.5 \mu$ s proton $90^{\circ}$ pulse, $1.75 \mathrm{~ms} \mathrm{CP}$ contact time at $67.5 \mathrm{kHz}$ and $9 \mathrm{~s}$ recycle time. The number of acquisitions for the CP/MAS ${ }^{13} \mathrm{C}$ spectra was typically 5.120 scans. ${ }^{13} \mathrm{C}$ NMR spectra were referenced to the carbonyl peak of glycine at $176.03 \mathrm{ppm}$. All spectra were processed with Gaussian multiplication parameters of $\mathrm{LB}=-5 \mathrm{~Hz}$ and $\mathrm{GB}=0.1$.

From all NMR spectra, C4 regions were deconvoluted using Lorentzian lines for the crystalline part $(\mathrm{Cr}(\mathrm{I} \alpha)$ and $\mathrm{Cr}(\mathrm{I} \beta)$ ) and one Gaussian line for the less ordered cellulose (para-crystalline cellulose, PCr, accessible surfaces, AS, and inaccessible surface, IAS) (see Additional file 1: Table S1 and Fig. 5) [30, 41]. Signal at 81.74 ppm was assigned to hemicellulose ( $\mathrm{HC})$, more specifically xylan, and was deconvoluted into one Gaussian line. The cellulose crystallinity, measured as the crystallinity index $(\mathrm{CrI})$, was determined from the areas of the 
crystalline $\left(A_{\text {cryst }}, 86-92 \mathrm{ppm}\right)$ and amorphous $\left(A_{\text {amorp}}\right.$, 78-86 ppm) C4 signals from spectral deconvolution as $\mathrm{CrI}=A_{\text {crys }} /\left(A_{\text {cryst }}+A_{\text {amorp }}\right) \times 100 \%$.

\section{Additional file}

Additional file 1: Fig S1. Monitoring of the recombinant production of PaLPMO9E in a $1.3 \mathrm{~L}$ bioreactor. Fig $\mathbf{S 2}$ a Image of the enzymatic treatment of birchwood Kraft fibers in the Tornado multiple chamber reactor. The enzymatic treatment was performed in parallel in four chambers using $7 \mathrm{~g}$ of cellulose fibers in each of them. $\mathbf{b}$ Aspect of the PaLPMO9Etreated bleached birchwood Kraft fibers after filtration. Fig S3 a HPAEC chromatograms showing the elution profile of oxidized and non-oxidized oligosaccharides and $\mathbf{b}$ quantification by HPAEC analysis of the soluble sugars released by the action of PaLPMO9E on starting bleached birchwood Kraft fibers (KF), PaLPMO9E-treated bleached birchwood Kraft fibers (KF-LPMO), Ultra Turrax dispersed PaLPMO9E-treated fibers (NFCO) and PaLPMO9E-treated fibers submitted to mechanical shearing (NFC1-3). Fig S4 Normalized HPSEC-RI chromatograms of solubilized fibers from starting bleached birchwood Kraft fibers (KF), PaLPMO9E-treated bleached birchwood Kraft fibers (KF-LPMO), Ultra Turrax dispersed PaLPMO9Etreated fibers (NFCO) and PaLPMO9E-treated fibers submitted to mechanical shearing (NFC1-3). Table S1. Characteristics (chemical shift position of the peaks, $\delta$, and full width at half height, FWHH) of signals generated from the deconvolution at the $\mathrm{C}-4$ region of the solid-state ${ }^{13} \mathrm{C} C \mathrm{CP} / \mathrm{MAS}$ NMR spectra of starting bleached birchwood Kraft fibers (KF), PaLPMO9Etreated bleached birchwood Kraft fibers (KF-LPMO), Ultra Turrax dispersed PaLPMO9E-treated fibers (NFCO) and PaLPMO9E-treated fibers submitted to mechanical shearing (NFC1-3).

\section{Abbreviations}

AFM: atomic force microscopy; CP/MAS: cross-polarization/magic angle spinning; HPAEC-PAD: high-performance anion exchange chromatography coupled with pulsed amperometric detection; HPSEC: high-performance size exclusion chromatography; KF: bleached birchwood Kraft fibers; KF-LPMO: bleached birchwood Kraft fibers treated by PaLPMO9E; LPMO: Iytic polysaccharide monooxygenase; MALLS: multi-angle laser light scattering; $M_{n}$ : number average molar mass; $M_{\text {w: }}$ : weight average molar mass; NFC: nanofibrillated cellulose; NFCO: bleached birchwood Kraft fibers treated by PaLPMO9E and submitted to Ultra Turrax dispersion; NFC1-3: bleached birchwood Kraft fibers treated by PaLPMO9E and submitted to mechanical shearing; PASC: phosphoric acid swollen cellulose; Rl: differential refractometer; TEMPO: 2,2,6,6-tetramethylpiperidine-1-oxyl.

\section{Acknowledgements}

The authors acknowledge the BIBS platform of INRA Angers-Nantes for providing access to microscopy (AFM) and NMR facilities. We greatly thank Nadège Beury for her excellent technical support for the AFM and optical microscopy images.

\section{Authors' contributions}

IG, SG and JGB produced the enzyme in bioreactor and purified the enzyme, performed the enzymatic treatment and analyzed soluble sugars; STL, VM and MPC performed mechanical treatments; CM, SLG, BC and AV characterized the fibers. All authors analyzed the data. All authors read and approved the final manuscript.

\section{Funding}

Region Pays de la Loire (Project Nanomach, Etoiles Montantes 2017-10691).

\section{Availability of data and materials}

All data generated or analyzed during this study are included in this published article and its additional information files.

\section{Ethics approval and consent to participate}

Not applicable.

\section{Consent for publication}

All authors read and approved the final manuscript.

\section{Competing interests}

The authors declare that they have no competing interests.

\section{Author details}

1 UR1268 Biopolymères Interactions Assemblages, INRA, 44316 Nantes, France. ${ }^{2}$ InTechFibres Division, FCBA, Domaine Universitaire, CS 90252, 39044 Grenoble Cedex 9, France. ${ }^{3}$ Biodiversité et Biotechnologie Fongiques, INRA, Aix Marseille University, UMR1 163, 13009 Marseille, France. ${ }^{4}$ CTP, Domaine Universitaire, CS 90252, 39044 Grenoble Cedex 9, France.

Received: 1 February 2019 Accepted: 15 June 2019

Published online: 24 June 2019

\section{References}

1. Jiang F, Li T, Li YJ, Zhang Y, Gong A, Dai JQ, Hitz E, Luo W, Hu LB. Wood-based nanotechnologies toward sustainability. Adv Mater. 2018:30:1703453.

2. Mika LT, Csefalvay E, Nemeth A. Catalytic conversion of carbohydrates to initial platform chemicals: chemistry and sustainability. Chem Rev. 2018;118:505-613.

3. Habibi Y, Lucia LA, Rojas OJ. Cellulose nanocrystals: chemistry, self-assembly, and applications. Chem Rev. 2010;110:3479-500.

4. Isogai A. Wood nanocelluloses: fundamentals and applications as new bio-based nanomaterials. J Wood Sci. 2013;59:449-59.

5. Klemm D, Kramer F, Moritz S, Lindström T, Ankerfors M, Gray D, Dorris A. Nanocelluloses: a new family of nature-based materials. Angew Chem Int Ed. 2011;50:5438-66.

6. Lavoine N, Desloges I, Dufresne A, Bras J. Microfibrillated cellulose-its barrier properties and applications in cellulosic materials: a review. Carbohydr Polym. 2012;90:735-64.

7. Moreau C, Villares A, Capron I, Cathala B. Tuning supramolecular interactions of cellulose nanocrystals to design innovative functional materials. Ind Crops Prod. 2016;93:96-107.

8. Nechyporchuk O, Belgacem MN, Bras J. Production of cellulose nanofibrils: a review of recent advances. Ind Crops Prod. 2016;93:2-25.

9. Rånby BG. Fibrous macromolecular systems. Cellulose and muscle. The colloidal properties of cellulose micelles. Discuss Faraday Soc. 1951:11:158-64.

10. Rånby BG. Physico-chemical investigations on animal cellulose (Tunicin). Arkiv Kemi. 1952;4:241-8.

11. Turbak A, Snyder F, Sandberg K. Microfibrillated cellulose, a new cellulose product: properties, uses, and commercial potential. J Appl Polym Sci. 1983;37:815-27.

12. Herrick FW, Casebier RL, Hamilton JK, Sandberg KR. Microfibrillated cellulose: morphology and accessibility. J Appl Polym Sci. 1983;37:797-813.

13. Azizi Samir MAS, Alloin F, Dufresne A. Review of recent research into cellulosic whiskers, their properties and their application in nanocomposite field. Biomacromolecules. 2005;6:612-26.

14. Capron I, Rojas OJ, Bordes R. Behavior of nanocelluloses at interfaces. Curr Opin Colloid Interface Sci. 2017;29:83-95.

15. Kalashnikova I, Bizot H, Cathala B, Capron I. New Pickering emulsions stabilized by bacterial cellulose nanocrystals. Langmuir. 2011;27:7471-9.

16. Olivier C, Moreau C, Bertoncini P, Bizot H, Chauvet O, Cathala B. Cellulose nanocrystal-assisted dispersion of luminescent single-walled carbon nanotubes for layer-by-layer assembled hybrid thin films. Langmuir. 2012:28:12463-71.

17. Mougel JB, Adda C, Bertoncini P, Capron I, Cathala B, Chauvet O. Highly efficient and predictable noncovalent dispersion of single-walled and multi-walled carbon nanotubes by cellulose nanocrystals. J Phys Chem C. 2016;120:22694-701.

18. Saito T, Nishiyama Y, Putaux J-L, Vignon M, Isogai A. Homogeneous suspensions of individualized microfibrils from TEMPO-catalyzed oxidation of native cellulose. Biomacromolecules. 2006;7:1687-91.

19. Saito T, Kimura S, Nishiyama Y, Isogai A. Cellulose nanofibers prepared by TEMPO-mediated oxidation of native cellulose. Biomacromolecules. 2007;8:2485-91. 
20. Paakko M, Ankerfors M, Kosonen H, Nykanen A, Ahola S, Osterberg M, Ruokolainen J, Laine J, Larsson PT, Ikkala O, et al. Enzymatic hydrolysis combined with mechanical shearing and high-pressure homogenization for nanoscale cellulose fibrils and strong gels. Biomacromolecules. 2007:8:1934-41.

21. Wagberg L, Decher G, Norgren M, Lindstroem T, Ankerfors M, Axnaes K. The build-up of polyelectrolyte multilayers of microfibrillated cellulose and cationic polyelectrolytes. Langmuir. 2008;24:784-95.

22. Nie SX, Zhang K, Lin XJ, Zhang CY, Yan DP, Liang HM, Wang SF. Enzymatic pretreatment for the improvement of dispersion and film properties of cellulose nanofibrils. Carbohydr Polym. 2018;181:1136-42.

23. Long LF, Tian D, Hu JG, Wang F, Saddler J. A xylanase-aided enzymatic pretreatment facilitates cellulose nanofibrillation. Bioresour Technol. 2017;243:898-904.

24. Lecourt M, Sigoillot JC, Petit-Conil M. Cellulase-assisted refining of chemical pulps: impact of enzymatic charge and refining intensity on energy consumption and pulp quality. Process Biochem. 2010;45:1274-8.

25. Lecourt M, Meyer V, Sigoillot JC, Petit-Conil M. Energy reduction of refining by cellulases. Holzforschung. 2010;64:441-6.

26. Tandrup T, Frandsen KEH, Johansen KS, Berrin J-G, Lo Leggio L. Recent insights into lytic polysaccharide monooxygenases (LPMOs). Biochem Soc Trans. 2018;46:1431-47.

27. Johansen KS. Discovery and industrial applications of lytic polysaccharide mono-oxygenases. Biochem Soc Trans. 2016;44:143-9.

28. Valenzuela SV, Valls C, Schink V, Sánchez D, Roncero MB, Diaz P, Martínez J, Pastor FIJ. Differential activity of lytic polysaccharide monooxygenases on celluloses of different crystallinity. Effectiveness in the sustainable production of cellulose nanofibrils. Carbohydr Polym. 2019;207:59-67.

29. Hu J, Tian D, Renneckar S, Saddler JN. Enzyme mediated nanofibrillation of cellulose by the synergistic actions of an endoglucanase, lytic polysaccharide monooxygenase (LPMO) and xylanase. Sci Rep. 2018;8:3195.

30. Villares A, Moreau C, Bennati-Granier C, Garajova S, Foucat L, Falourd X, Saake B, Berrin JG, Cathala B. Lytic polysaccharide monooxygenases disrupt the cellulose fibers structure. Sci Rep. 2017;7:40262.

31. Ladeveze S, Haon M, Villares A, Cathala B, Grisel S, Herpoel-Gimbert I, Henrissat B, Berrin JG. The yeast Geotrichum candidum encodes functional lytic polysaccharide monooxygenases. Biotechnol Biofuels. 2017;10:215.

32. Bennati-Granier C, Garajova S, Champion C, Grisel S, Haon M, Zhou S, Fanuel M, Ropartz D, Rogniaux H, Gimbert I, et al. Substrate specificity and regioselectivity of fungal AA9 lytic polysaccharide monooxygenases secreted by Podospora anserina. Biotechnol Biofuels. 2015;8:90.

33. Chinga-Carrasco G. Cellulose fibres, nanofibrils and microfibrils: the morphological sequence of MFC components from a plant physiology and fibre technology point of view. Nanoscale Res Lett. 2011;6:417.

34. Siqueira G, Tapin-Lingua S, Bras J, da Perez DS, Dufresne A. Morphological investigation of nanoparticles obtained from combined mechanical shearing, and enzymatic and acid hydrolysis of sisal fibers. Cellulose. 2010;17:1147-58.

35. Scheller HV, Ulvskov P. Hemicelluloses. Annu Rev Plant Biol. 2010;61:263-89.

36. Hoebler C, Barry JL, David A, Delortlaval J. Rapid acid hydrolysis of plant cell wall polysaccharides and simplified quantitative determination of their neutral monosaccharides by gas-liquid chromatography. J Agric Food Chem. 1989;37:360-7.

37. Potthast A, Radosta S, Saake B, Lebioda S, Heinze T, Henniges U, Isogai A, Koschella A, Kosma P, Rosenau T, et al. Comparison testing of methods for gel permeation chromatography of cellulose: coming closer to a standard protocol. Cellulose. 2015;22:1591-613.

38. Hasani M, Henniges U, Idstrom A, Nordstierna L, Westman G, Rosenau T, Potthast A. Nano-cellulosic materials: the impact of water on their dissolution in DMAc/LiCl. Carbohydr Polym. 2013;98:1565-72.
39. Couturier M, Ladeveze S, Sulzenbacher G, Ciano L, Fanuel M, Moreau C, Villares A, Cathala B, Chaspoul F, Frandsen KE, et al. Lytic xylan oxidases from wood-decay fungi unlock biomass degradation. Nat Chem Biol. 2018;14:306.

40. Zuckerstätter G, Schild G, Wollboldt P, Röder T, Weber HK, Sixta H. The elucidation of cellulose supramolecular structure by ${ }^{13} \mathrm{C}$ CP-MAS NMR. Lenzing Ber. 2009;87:38-46.

41. Larsson PT, Wickholm K, Iversen T. A CP/MAS C-13 NMR investigation of molecular ordering in celluloses. Carbohydr Res. 1997;302:19-25.

42. Liitiä T, Maunu SL, Hortling B, Tamminen T, Pekkala O, Varhimo A. Cellulose crystallinity and ordering of hemicelluloses in pine and birch pulps as revealed by solid-state NMR spectroscopic methods. Cellulose. 2003;10:307-16.

43. Peciulyte A, Karlstoem K, Larsson PT, Olsson L. Impact of the supramolecular structure of cellulose on the efficiency of enzymatic hydrolysis. Biotechnol Biofuels. 2015;8:56.

44. Malm E, Bulone V, Wickholm K, Larsson PT, Iversen T. The surface structure of well-ordered native cellulose fibrils in contact with water. Carbohydr Res. 2010;345:97-100.

45. Wagberg L, Winter L, Odberg L, Lindstrom T. On the charge stoichiometry upon adsorption of a cationic polyelectrolyte on cellulosic materials. Colloids Surf. 1987;27:163-73.

46. Cathala B, Villares A, Moreau C, Berrin JG. Procedure for the fabrication of nanocellulose from a cellulosic substrate. French patent FR 2015/1555049. 2015.

47. Besbes I, Vilar MR, Boufi S. Nanofibrillated cellulose from Alfa, Eucalyptus and Pine fibres: preparation, characteristics and reinforcing potential. Carbohydr Polym. 2011;86:1198-206.

48. Chaker A, Mutje P, Vilaseca F, Boufi S. Reinforcing potential of nanofibrillated cellulose from nonwoody plants. Polym Compos. 2013;34:1999-2007.

49. Simmons TJ, Mortimer JC, Bernardinelli OD, Poppler AC, Brown SP, Deazevedo ER, Dupree R, Dupree P. Folding of xylan onto cellulose fibrils in plant cell walls revealed by solid-state NMR. Nat Commun. 2016;7:13902.

50. Wang WX, Sabo RC, Mozuch MD, Kersten P, Zhu JY, Jin YC. Physical and mechanical properties of cellulose nanofibril films from bleached eucalyptus pulp by endoglucanase treatment and microfluidization. J Polym Environ. 2015;23:551-8.

51. Couturier M, Haon M, Coutinho PM, Henrissat B, Lesage-Meessen L, Berrin JG. Podospora anserina hemicellulases potentiate the Trichoderma reesei secretome for saccharification of lignocellulosic biomass. Appl Environ Microbiol. 2011;77:237-46.

52. Westereng B, Agger JW, Horn SJ, Vaaje-Kolstad G, Aachmann FL, Stenstrom YH, Eijsink VGH. Efficient separation of oxidized cello-oligosaccharides generated by cellulose degrading lytic polysaccharide monooxygenases. J Chromatogr A. 2013;1271:144-52.

53. Horcas I, Fernandez R, Gomez-Rodriguez JM, Colchero J, Gomez-Herrero J, Baro AM. WSXM: A software for scanning probe microscopy and a tool for nanotechnology. Rev Sci Instrum. 2007;78:013705.

54. Blakeney AB, Harris PJ, Henry RJ, Stone BA. A simple and rapid preparation of alditol acetates for monosaccharide analysis. Carbohydr Res. 1983;113:291-9.

\section{Publisher's Note}

Springer Nature remains neutral with regard to jurisdictional claims in published maps and institutional affiliations. 